\title{
The Virtual Screening to Search Proplasmepsin II Inhibitor from Indonesian Medicinal Plant Phytochemicals: Anti-Malaria
}

\author{
Muhammad Habiburrohman, Wilda Nur Rohmatilah, Arthur Hariyanto Prakoso, \\ Bawon Triatmoko, Ari Satia Nugraha*
}

Drug Utilisation and Discovery Research Group, Faculty of Pharmacy, University of Jember, Jember, Indonesia 68121

*Corresponding author:

E-mail: arisatia@unej.ac.id

\begin{abstract}
The indigenous people of Indonesia have used medicinal plants to survive infectious diseases, including malaria. The knowledge has been passed through the generation and a limited number of the plants have been studied properly. Malaria infection has been an endemic and complicated problem in the archipelago where drug-resistant cases worsen the situation. Natural product study without pharmacological information has been a drawback to the development of natural-based antimalarial. Computational chemistry protocol gives lower cost facilitation to later a conventional in vitro bioassay. In this study, virtual screening was deployed to find a Proplasmepsin II enzyme inhibitor, in which the enzyme plays an important in this parasite metabolism. The enzyme (1PFZ) was collected from the PDB database followed by docking validation before exhaustive docking calculation of 238 compounds from 43 Indonesian medicinal plants. The docking protocol was valid as indicated by rmsd value of $1.275 \AA$. One top hit molecule, AM56, was gained with its free energy of binding value of $10.8 \mathrm{kcal} / \mathrm{mol}$ which is better than the interaction of the native ligand, propane-1,2,3-Triol, with the free energy of binding $(\Delta \mathrm{G})$ score of $3.9 \mathrm{kcal} / \mathrm{mol}$. AM56 was a secondary metabolite of Borassus flabellifer and its anti-plasmodium was previously studied. However, the mechanism of action of AM56 was never been reported. This study was able to discover a molecular scaffold of proplasmepsin II enzyme inhibitor and can be used as a pathway to QSAR study of AM56 semi-synthetic derivatives.
\end{abstract}

Keywords: Molecular docking, plasmodium, antimalaria, Proplasmepsin II Inhibitor, 1PFZ

\section{Introduction}

Indonesia is a tropical land that has a high prevalence of malaria. The Annual Parasite Incidence (API) is the number of new infections per year per 1000 population. This is evidenced by data from the Indonesia Ministry of Health, a total of the city that have API $<1$ and can raised the elimination of malaria in every province 2018. From 34 provinces in Indonesia, five provinces are not yet declared free from malaria, including Papua (Annual Parasite Incidence, API, 16), East Nusa Tenggara (API 5), Maluku (API 5), North Maluku (API 2), and East Borneo (API 1) (Pudjiastuti et al., 2014).

Geographically, the endemic areas are usually remote. This area has limited health access, so people in that place survive from malaria by using traditional medicinal plants. We know that many studies discover and develop medicinal plants to search for a new drug for malaria. But many questions remain about their efficacy and safety. Malaria is caused by Plasmodium sp. This

How to cite:

Habiburrohman, M., Rohmatillah, W. N., Prakoso, A. H., Triatmoko, B., \& Nugraha, A, S. (2021). The virtual screening to search proplasmepsin II Inhibitor from Indonesian medicinal plant phytochemicals: Anti-malaria. The 3rd International Conference on Life Sciences and Biotechnology. NST Proceedings. pages 25-30. doi: 10.11594/ nstp.2020.0804 
parasite is transmitted by Anopheles sp. mosquitos. One way for this parasite to infect humans is to digest the hemoglobin.

The parasites cause disease during the Intraerythrocytic phase and consume nearly all the hemoglobin of the human host to generate amino acids for growth and maturation. Hemoglobin is degraded by a series of proteases. Hemoglobin is degraded by a series of proteases in an acidic digestive vacuole of the parasite. The aspartic proteases found in the vacuole, named plasmepsin, make an initial attack on the hemoglobin molecule, followed by proteolysis of the large fragments into small peptides by a cysteine protease named falciparum (Asojo et al., 2013).

One of the plasmepsins that can digest hemoglobin is Plasmepsin II. An efficient method of production of active recombinant plasmepsin II employed a truncated form of proplasmepsin II, in which the first 76 amino acids of the prosegment were missing (Bernstein \& James, 1999). This research aims to find a potent compound -from a medicinal plant- that can inhibit proplamepsi II.

\section{Material and Methods \\ Materials}

Hardware: The author use PC Linux Ubuntu 18.0, Intel $®$ Xeon $®$ to process molecular docking. Software: ChemDraw ultra 12 to draw target compound, Chem $3 \mathrm{~d}$ to visualization, and to minimize energy compound. Autodock vina to docking, and Pymol to visualization. The author get the enzyme from rcsb.org

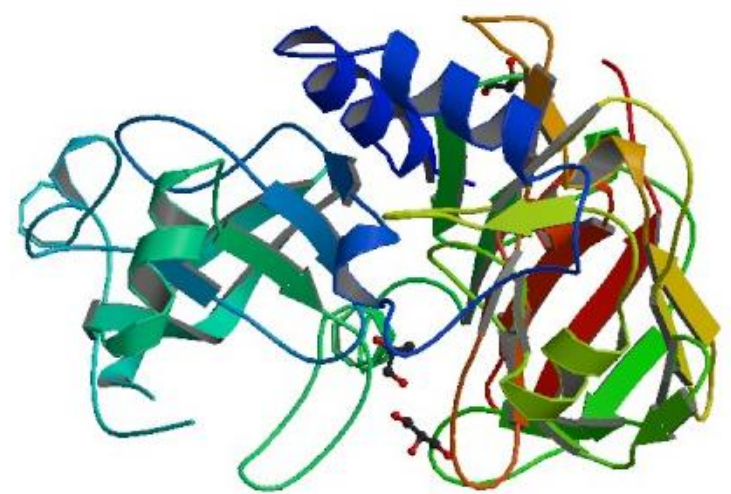

Figure 1. Actinomycetes isolates growth in HV agar media

\section{Enzyme Preparation}

Proplasmepsin II was downloaded from the protein data bank (www.rcsb.org) with codenamed 3FNT and through Chem3D 12 all the water molecules were removed. The enzyme was separated from the original ligand using the PyMOL. Then the enzyme was prepared by adding polar hydrogen using autodock tools. The target enzyme was ready for the molecular docking process.

\section{Ligand Preparation}

The native ligands and 238 compounds selected from the list of antimalarial compounds were drawn in 2D and energy minimized using the MM2 method and saved in the PDB file extension. The method used in minimizing energy was the 'force field molecular mechanism MM2' method. Then the ligand was prepared through autodock tools and save as a.PDBQT file extension. Griding and Molecular Docking.

\section{Griding}

Gridding is used to determine the right region enzyme for docking. In this study, we used the original ligand as a reference for the ligand dock. This process uses autodock tools. 


\section{Docking molecular}

The molecular docking process is used autodock vina software. Enzyme and ligand files with the extension .pdbqt are saved in one folder as many as 238 folders according to the number of compounds. Then added "conf.txt", a text with the extension .txt containing the file name, and the gridding region of the position attached to the ligand.

\section{Visualization}

The docked ligands then visualized using the Pymol software. This software can read various enzyme and ligand extensions. Also, from the Pymol software, interactions between the ligand and its enzyme are obtained in free energy of binding $(\Delta G)$ value.

\section{Results and Discussion}

Molecular docking is a computational procedure that attempts to predict the noncovalent binding of macromolecules or, more frequently, of a macromolecule (receptor) and a small molecule (ligand) efficiently, starting with their unbound structures, structures obtained from MD simulations, or homology modeling, etc. The goal is to predict the bound conformations and the binding affinity.

To validate the method, we docked a native ligand to the enzyme. The parameters used for this validation are free energy of binding and Root Mean Square Deviation (RMSD). Free energy of binding score is used to be a comparator target compound. The good RMSD is $<2 \AA$, so the confirmation of the ligand after docked with the native ligand does not deviate too far. We obtained native ligand's free energy of binding $(\Delta \mathrm{G})$ of $-3.7 \mathrm{kcal} / \mathrm{mol}$ and RMSD $0.775 \AA$.

The parameters used in the docking process are the free energy of binding $(\Delta G)$ as a marker of affinity. The more negative $\Delta G$ shows higher ligand affinity for the active site of the receptor. Affinity shows how strong the bonding between ligand and receptor. The higher the affinity is, the stronger the bonding between ligand and receptor is a compound -ligand - that has high affinity is potent to be used to the drug. Because the ligand with affinity can shift the native ligand (with lower affinity). So, it can make an effect after bonding with the receptor.

In thermodynamic, plus (+) or minus (-) signs show the direction. The plus shows that the reaction is endotherm and in that reaction need some energy (heat). While minus (-) shows that the reaction is exothermic where the reaction releases energy (heat). The good reaction is trend release energy (no to need the energy). The endothermic reaction happens when there are heat was absorbed (needed) to react, while the exothermic reaction happens when the heat is released to the surrounding. So, when an exothermic reaction happens, $\Delta \mathrm{G}$ will be negative, the affinity is high and then the complex of ligand-receptor is easier to be formed. This is a scheme receptor occupancy theory (Boss et al., 2003):

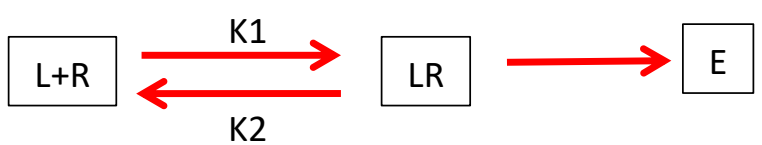

$$
\mathrm{L}=\text { ligand } ; \mathrm{R}=\text { receptor } ; \mathrm{E}=\text { efect }
$$

K1 is concentration that show how many ligand occupy receptor. If K1 high, concentration ligand that occupies receptor is high too, so the intensity of effect is bigger. On the otherwise, K2 is concentration that show how many ligand detached (or not bonding) from receptor. If K2 high, concentration of ligand that occupy receptor is low, so the intensity of effect is lower. Ratio of K1 and $\mathrm{K} 2$ is called affinity. To get high bond between ligand and receptor, which it show high intensity effect, we can see it from free energy of binding $(\Delta G)$ score. 
Table 1. Contain compounds and its specification

$\begin{array}{lll}\begin{array}{ll}\text { Compound } \\ \text { code }\end{array} & \begin{array}{l}\text { Plant } \\ \text { source }\end{array} & \begin{array}{l}\text { Ligand's two-dimension structures and its } \\ \text { name }\end{array}\end{array}$

Native lig- -

and<smiles>OCC(O)CO</smiles>

\section{(Glycerol)}

Borassus

AM 56

flabellifer

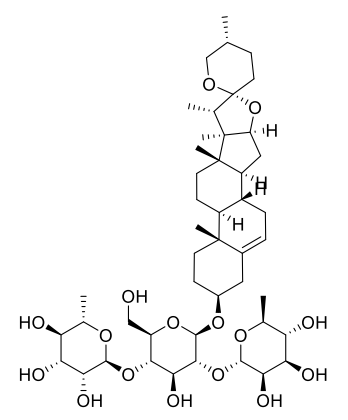

$-10,8$

(Dioscin)

AM51

Borassus

flabellifer

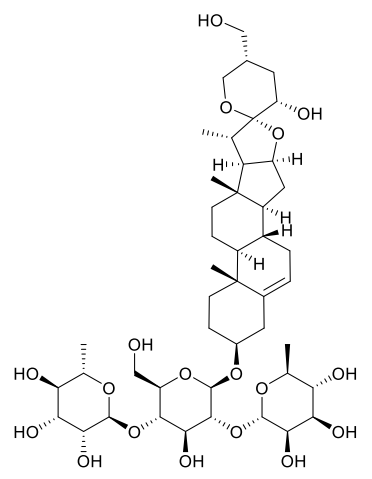

(Borassosides B)

AM50
Borassus
flabellifer

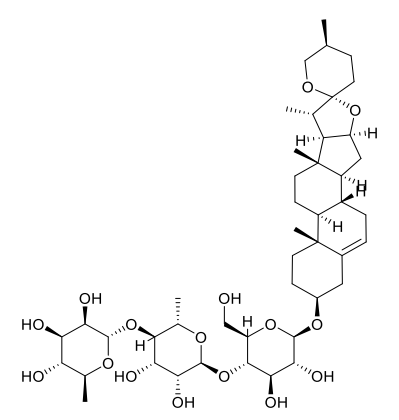

$-10.2$

(Borassosides A)
$-10.5$ 
Table 2. Amino acid residues

\section{Ligand}

Native ligand (Glycerol)

AM56

AM51

AM50

\section{Amino acid residues}

GLN-12,ASN-13,MET-15,LEU-33

ASN-328, HIS-161, HIS-276, GLN-275, LYS-163, ARG-176

ARG-176, THR81P, LYS-163, HIS-161,HIS-276, GLU-271

LEU-274, LYS327,HIS-161

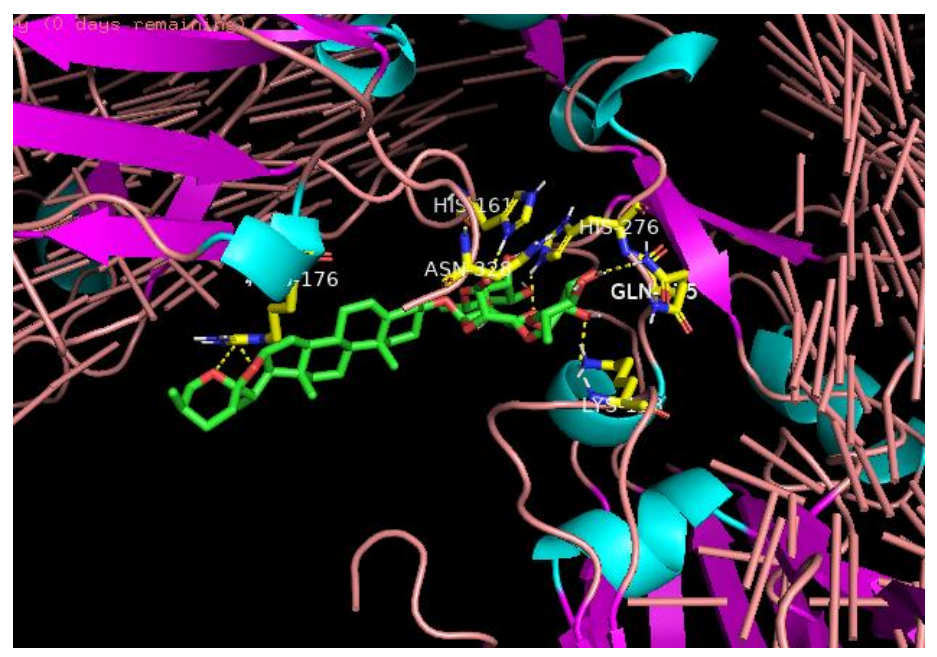

Figure 2. Interaction visualization AM56 against Proplasmepsin II showing hydrogen bonding with ASN328, HIS-161, HIS-276, GLN-275, LYS-163, ARG-176

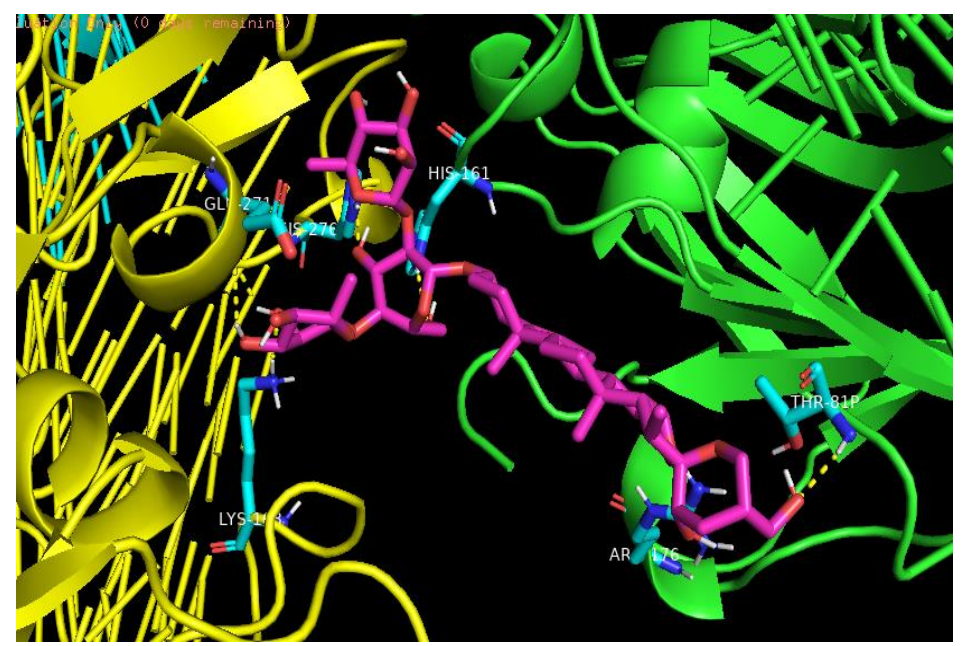

Figure 3. Interaction visualization AM51 against Proplasmepsin II showing hydrogen bonding with ARG176, THR81P, LYS-163, HIS-161, HIS-276, GLU-271 


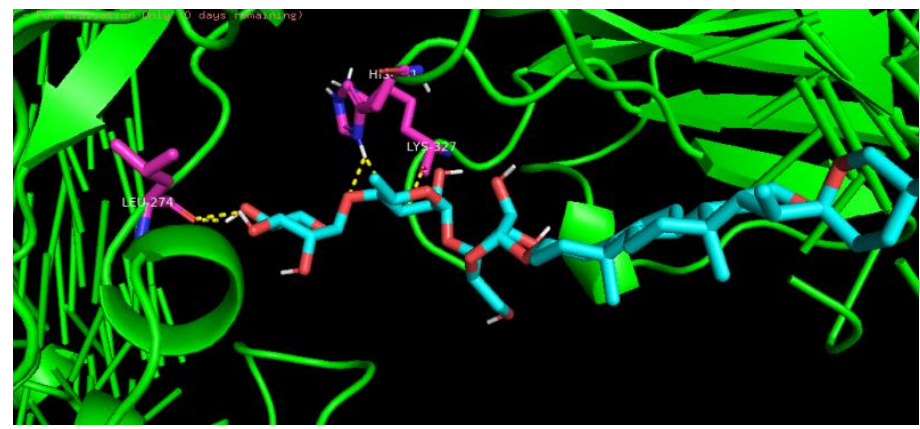

Figure 4. Interaction visualization AM50 against Proplasmepsin II showing hydrogen bonding with LEU-

274, LYS327, HIS-161

\section{Conclusion}

In conclusion, based on experimental results, this study succeeded in getting the three best compounds from Borassus flabellifer which have a great potential activity to inhibit proplasamepsin II. The highest affinity was shown by AM56 compounds. This compound has the best affinity with the free energy of binding $(\Delta G)$ score of $-10.8 \mathrm{kcal} / \mathrm{mol}$. Compared with the native ligand (glycerol) with a score of $-3.7 \mathrm{kcal} / \mathrm{mol}$. Thus, these three compounds can be an alternative to get potential antimalarial candidates from medicinal plants. Also, it is necessary to do both in vitro and in vivo tests to prove the HAP inhibition activity of the three candidate compounds on Plasmodium falciparum

\section{References}

Asojo, O. A., Gulnik, S. V., Afonina, E., Yu, B., Ellman, J. A., Haque, T.S., \& Silva, A. M. (2013). Novel Uncomplexed and complexed structures of plasmepsin II, an Aspartic protease from Plasmodium falciparum. Journal of Molecular Biology, 327(1), 173-81. doi: 10.1016/s0022-2836(03)00036-6

Bernstein, N. K., Cherney, M.M., Loetscher, H., Ridley, R.G., \& James, M.N. (1999). Crystal structure of the novel aspartic proteinase zymogen proplasmepsin II from Plasmodium falciparum. Natural Structural \& Molecular Biology, 6(1), 32-7. doi: 10.1038/4905.

Bernstein, N.K., \& James, M.N.G. (1999). Novel ways to prevent proteolysis — prophytepsin and proplasmepsin II. Current Opinion in Structural Biology, 9(6), 690-5. doi: 10.1016/s0959-440x(99)00030-5

Boss, C., Richard-Bildstein, S., Weller, T., Fischli, W., Meyer, S., \& Binkert, C. (2003). Inhibitors of the Plasmodium falciparum parasite aspartic protease plasmepsin II as potential antimalarial agents. Current Medicinal Chemistry, 10(11), 883-907. doi: 10.2174/0929867033457674

Brain, S. D. (1992). Medical Pharmacology at a Glance. Cardiovascular Research, 26, 1227

Pudjiastuti, P., Sumarsih, S., Arwati, H., Amalina, I., Fanani, M. Z., Utomo, E. P., Fitri, L.E., Nugraha, A. S., Lie, W., \& Pyne, S.G. (2014). Epicroomine and croomine from Stemona tuberosa Antimalarial drug for inhibiting dihydrofolate reductase (DHFR) Activity and their molecular modelling. Journal of Chemical and Pharmaceutical Research, 6, 544-8 